\title{
Distribución y abundancia de la ascidia Ecteinascidia turbinata (Ascidiacea: Perophoridae) en Cuba
}

\author{
Aida Hernández-Zanuy ${ }^{1}$, José Luis Carballo ${ }^{2}$, Alida García-Cagide ${ }^{1}$, Santiago Naranjo ${ }^{3}$ \& \\ Macario Esquivel ${ }^{1}$ \\ 1 Instituto de Oceanología del Ministerio de Ciencia Tecnología y Medio Ambiente de Cuba. Ave. 1era y 186 No. \\ 18406, Ciudad Habana, Cuba aidah@oceano.inf.cu \\ 2 Instituto de Ciencias del Mar y Limnología, UNAM. Mazatlán. A.P.811. Mazatlán 82000. México.FAX: \\ 5269826133JLjlcarballo@ola.icmyl.unam.mx \\ 3 Departamento de Biología Marina. Pharma-Mar S.A. Tres Cantos. 2876. Madrid, España.
}

Recibido 10-VII-2002. Corregido 31-I-2006. Aceptado 06-XI-2006.

\begin{abstract}
Distribution and abundance of the ascidian Ecteinascidia turbinata (Ascidiacea: Perophoridae) in Cuba. Permanently submerged mangrove roots (Rhizophora mangle) are the main habitat of the ascidian Ecteinascidia turbinata in Cuba. It was occasionally found on black coral (Antiphates caribeana) between 22 and 38 meters deep. This species exhibits a wide distribution in all the mangrove keys surrounding the Island of Cuba but does not occur in riparian or fringing mangroves. Populations of this species are abundant in Cuba: in $75 \%$ of the 58 localities sampled the species was present and in $57 \%$ more than $50 \%$ of the roots held at least one colony. The highest colony densities were found in the northern coast of Pinar del Río province with values near one colony per lineal meter of mangrove root. We found the highest density $(1.46 \mathrm{col} / \mathrm{m})$ and greatest biomass at Jutías Key, with values between 25 and $660 \mathrm{~g} / \mathrm{m}$. The average of wet biomass in the studied mangroves was $73.63 \mathrm{~g} / \mathrm{m}$. Rev. Biol. Trop. 55 (1): 247-254. Epub 2007 March. 31.
\end{abstract}

Key words: ascidian, Ecteinascidia turbinata, distribution, abundance, density, biomass, mangrove, Cuba.

La ascidia Ecteinascidia turbinata Herdman, 1880 tiene una distribución anfiatlántica en áreas tropicales y subtropicales, que abarca desde las costas españolas hasta el norte del Brasil (Naranjo 1995). En la región caribeña ha sido registrada en la mayoría de las islas antillanas y algunas localidades centroamericanas (Van Name 1921, 1945, Millar 1962, 1978, Monniot y Monniot 1984, Orihuela 1991, Littler 1997). En Cuba su presencia se reportó en Cayo Los Indios, Punta del Este y en la laguna arrecifal de Diego Pérez (Hernández-Zanuy 1990).

La especie se encuentra en todos los hábitats litorales, desde la zona de las mareas, hasta los arrecifes exteriores (Monniot 1972) y aunque habita preferentemente en los fondos someros, su límite batimétrico se encuentra en $42 \mathrm{~m}$ de profundidad (Pèrés 1956).

En los últimos años E. turbinata se ha estudiado intensamente debido a la presencia de compuestos con actividad anticancerígena (Rinehart et al. 1990, Sakai et al. 1992, Guan et al. 1993) y aunque han sido numerosos los intentos de síntesis química de estas sustancias (Kerr y Miranda 1995, Sakai et al. 1996, Saito et al. 1997) hasta la fecha la única vía de obtención de tales productos depende directamente de extracciones controladas a partir de las poblaciones naturales (Carballo et al. 1999), o potenciando su crecimiento sobre superficies artificiales (Carballo et al. 2000).

Con fines de investigación se extrajeron una tonelada de esta ascidia en la Florida y dos 
en el Caribe, produciéndose impactos negativos sobre las poblaciones naturales (Pain 1996). Sin embargo, posteriormente se han hecho grandes esfuerzos para conocer la biología de la especie tanto en el Caribe (Bingham and Young 1991a, 1995, Carballo 2000a, Hernández-Zanuy et al. 2000, Hernández-Zanuy 2003, García-Cagide et al. 2005,) como en el Mediterráneo (Carballo et al. 1997, Carballo 2000b), paso previo para una explotación de la misma a gran escala.

Debido a la gran abundancia de manglares que rodea las costas de Cuba es de suponer que E. turbinata tenga una amplia distribución en nuestro archipiélago, por lo que con el presente estudio se pretende conocer la distribución, la abundancia y el hábitat de E. turbinata en las costas de Cuba como base para su posible explotación como nuevo recurso marino.

\section{MATERIALES Y MÉTODOS}

Se muestrearon 58 localidades (Fig. 1, Cuadro 1) durante el verano de los años 1996 y 1998, inspeccionando visualmente las raíces de manglar Rhizophora mangle para detectar la presencia de E. turbinata. En cada estación se valoró la abundancia relativa según el porcentaje de raíces portadoras de al menos una colonia (se considera colonia al conjunto de dos o más zooides). La escala de abundancia utilizada fue: muy abundante (presente en más del $50 \%$ de las raíces), abundante (entre 25 y $50 \%)$, escasa $(<25 \%)$ y ausente $(0 \%)$.

Para estimar la densidad (colonias/metro lineal de manglar) y la biomasa $(\mathrm{g} / \mathrm{m})$, se seleccionó una localidad en cada uno de los tres subarchipiélagos de la plataforma cubana donde la especie estuviera presente (Punta del Este, Bahía de Cádiz y Norte de Pinar del Río) (Fig. 1). En cada una de ellas se seleccionaron al azar 6, 10 y 12 estaciones respectivamente (Cuadro 1), y en cada estación se estimó la densidad contando las colonias presentes a lo largo de una cuerda de $30 \mathrm{~m}$ de largo situada aleatoriamente a lo largo del margen del manglar. Para estimar la biomasa húmeda se cortaron pedazos de raíz portadoras de colonias, se colocaron individualmente en bolsas de plástico, y se transportaron al laboratorio, donde se

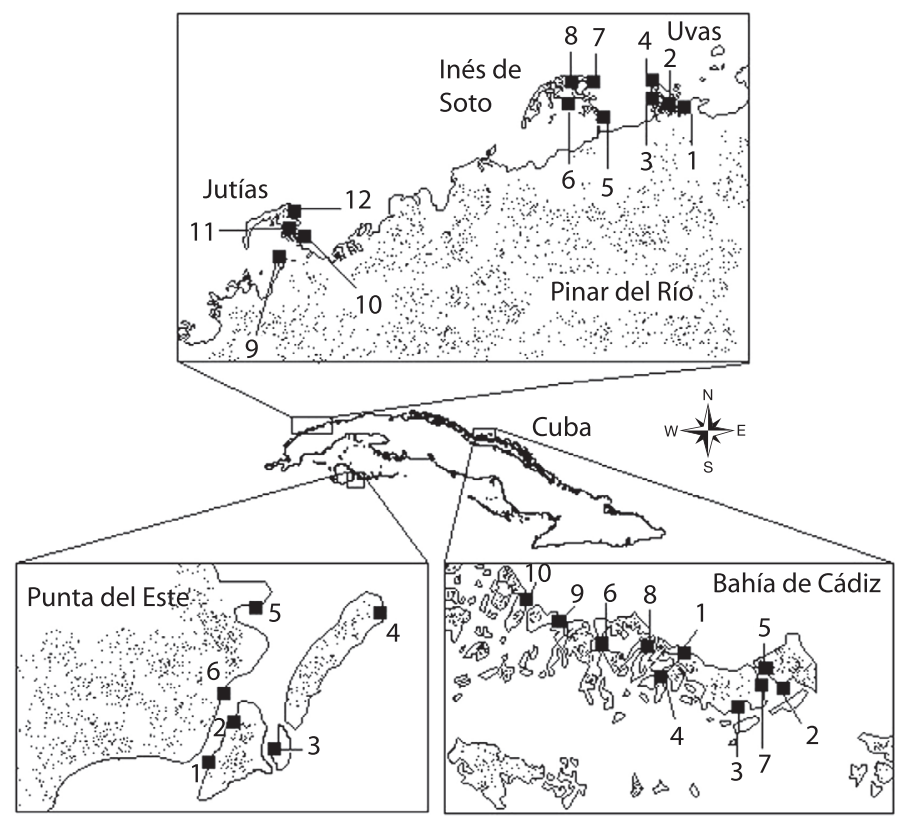

Fig. 1. Localización geográfica de las estaciones estudiadas: Norte de Pinar del Río, Punta del Este y Bahía de Cádiz. Fig. 1. Geografical location of the studied stations: Norte de Pinar del Río, Punta del Este y Bahía de Cádiz. 
limpiaron y pesaron en una balanza analítica (PRECISA XT 220A) con precisión $0.1 \mathrm{mg}$.

\section{RESULTADOS}

Hábitat: E. turbinata estuvo presente en las raíces sumergidas del mangle rojo de todos los manglares de cayo o manglar de tipo sobrelavado que permanecieran sumergidas a pesar de los cambios de marea. En una estación de Cayo Jutías se observaron numerosas colonias sobre la punta de los propágulos del mangle rojo que se disponen en posición vertical sobre los fondos muy someros de los canales entre los cayos. En la laguna de Santa Fé, al norte de la Habana, se observaron pequeñas colonias sobre las hojas de Thalassia testudinum Banks ex Konig. En la laguna del arrecife del Cayo Diego Pérez, al SO del Golfo de Batabanó se encontraron algunas colonias creciendo sobre los gorgonáceos muertos. Las poblaciones más numerosas se hallaron siempre en la zona litoral a unos pocos metros de profundidad, aunque se detectaron dos colonias de E. turbinata a 22 y $38 \mathrm{~m}$ de profundidad sobre corales negros (Anthipates caribeana). En ningún caso se observaron colonias sobre los sedimentos del fondo o superficies de algas. Fue muy frecuente la aparición de ejemplares de esta especie sobre esponjas y moluscos que conforman la epibiota de las raices sumergidas del mangle rojo e incluso sobre el carapacho de pequeños ejemplares del crustáceo Dromidea antillensis.

Distribución: la especie está presente de manera continua alrededor del archipiélago cubano (Fig. 2), siguiendo el patrón de distribución de los manglares de tipo sobrelavado (según la clasificación de Lugo y Snedaker (1974)). En la mitad noroccidental del país, E. turbinata se distribuye desde Médano de Casiguas, hasta Punta Tabacos, última localidad evaluada hacia el oeste. A partir de Médano de Casiguas hacia el este, sólo se encontró una pequeña población en un manglar de la localidad de Santa Fé. Continuando por la costa norte en la misma dirección, la especie reaparece en los manglares de Varadero y su presencia se mantiene hasta la Bahía de Vita en Holguín. En la mitad oriental del país, tanto en los cayos del norte como en los del sur, las poblaciones son poco densas y muy dispersas a pesar de existir una gran cantidad de manglares de cayo. En los cayos del Golfo de Batabanó (plataforma SO de Cuba), E. turbinata se encuentra ampliamente distribuida. En los cayos cercanos a la Ciénaga de Zapata sólo se detectó su presencia en la laguna arrecifal de Cayo Diego Pérez. En el Archipiélago de los Jardines de la Reina sólo se visitaron los cayos de mangle de la mitad occidental debido al mal estado de conservación en que se encuentra el resto de la cayería (según datos inéditos de Hernández-Zanuy). En los dos cayos visitados (Anclitas y Bretón) la especie está ampliamente distribuida. En los manglares de borde visitados (costa de tierra firme), sólo se detectó la presencia de la especie, en Punta del Este (Isla de la Juventud) y Santa Fé (Litoral Norte de la Habana).

Abundancia: la especie estuvo presente en el $75 \%$ de las localidades visitadas (Fig. 2). En el $25 \%$ resultó ser muy abundante (más del $50 \%$ de las raíces con E. turbinata), en el $32.14 \%$ de las estaciones era abundante (entre 25 y $50 \%$ de las raíces con E. turbinata) y en el $17.86 \%$ escasa (menos del $25 \%$ de las raíces). No se encontró en el $25 \%$ de las estaciones. En general las poblaciones de E. turbinata de los manglares de Cuba son abundantes pues en el $57 \%$ de las zonas prospectadas más del $25 \%$ de las raíces del manglar posee al menos una colonia. Tanto en Punta del Este como en Bahía de Cádiz, la densidad media de colonias no sobrepasa el valor de una colonia por cada dos metros de manglar recorridos. En la zona norte de Pinar del Río se encontraron las mayores densidades con valores cercanos a una colonia por metro lineal (Fig. 3), siendo Cayo Jutías la localidad que posee la mayor densidad (1.46 $\mathrm{col} / \mathrm{m})$. La biomasa húmeda en las estaciones estudiadas varió entre 3 y $660 \mathrm{~g} / \mathrm{m}$ (Fig. 4). Las colonias de mayor peso se encontraron en la costa Norte de Pinar del Río. En Cayo Jutías la biomasa obtenida osciló entre 25 y 660 g/m; 

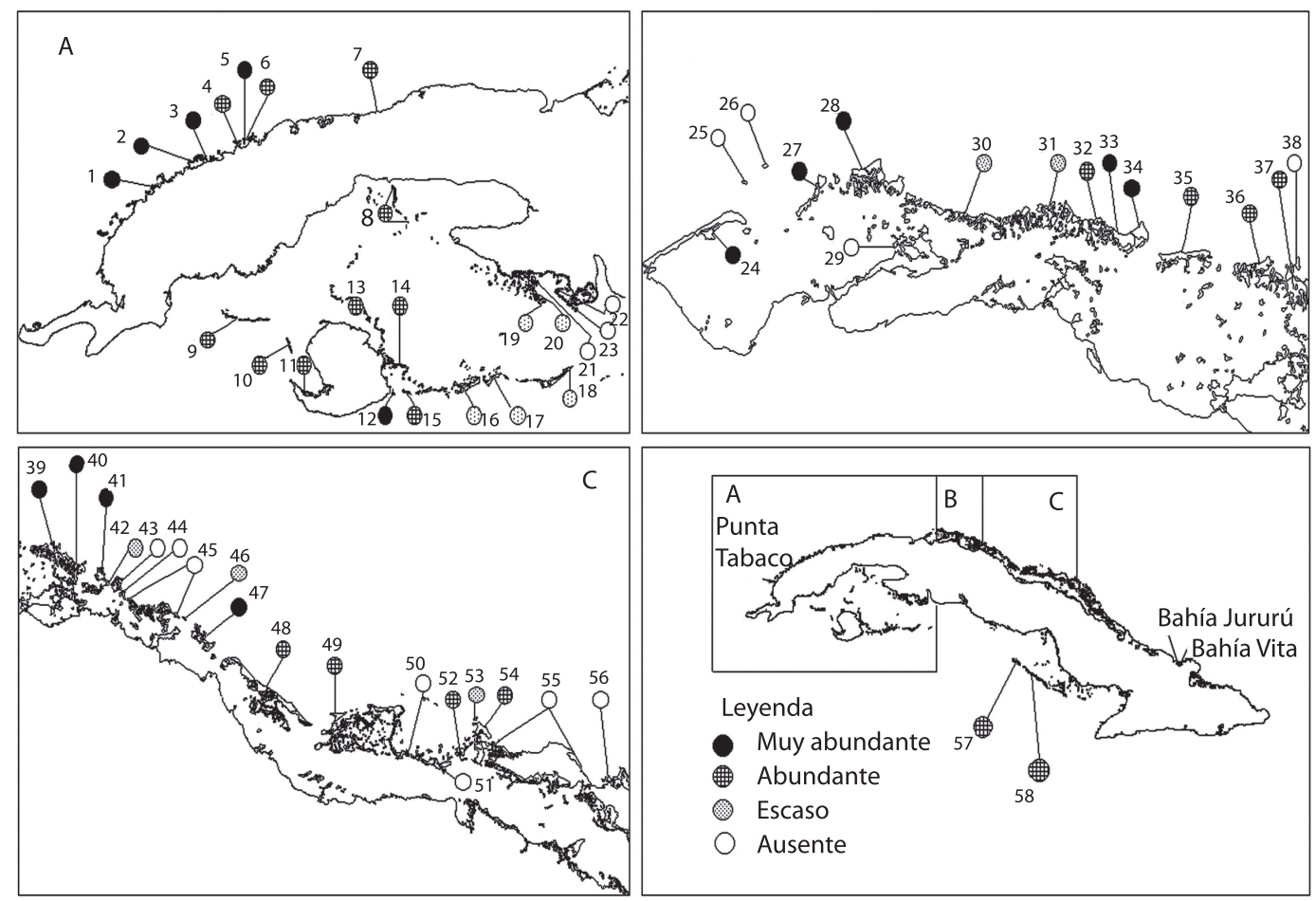

Fig. 2. Distribución y abundancia relativa de E. turbinata (\% de raíces con ascidias) en Cuba. 1: Cayo Jutías, 2: Cayo Inés de Soto, 3: Cayo Uvas, 4: Cayo Levisa, 5: Cayo Alacranes, 6: Cayo Médano de Casiguas, 7: Laguna de Santa Fé, 8: Cayería Las Cayamas, 9: Cayería San Felipe, 10. Cayo Los Indios, 11: Punta Piedras, 12: Punta del Este, 13: Cayo Los Inglesitos, 14: Cayo Bocas de Alonso, 15: Cayo Matías, 16: Cayo Cantiles, 17: Cayo Rosario, 18: Cayo Largo, 19: Cayo Flamenco, 20: Cayo Diego Pérez, 21: Cayo El Macío, 22: Cayo Calvario, 23: Cayo Ernest Thelman, 24: Canal de Chapulín, 25: Cayo Piedras del Norte, 26: Cayo Mono, 27: Cayos Blancos, 28: Cayo Cruz del Padre, 29: Cayo de las Cinco Leguas, 30: Cayo Ranas, 31: Canal Blanco, 32: Canal de la Cuberita, 33: Cayo Falcones, 34: Cayo Bahía de Cádiz, 35: Cayo Blaquizal, 36: Punta Sardines, 37: Bahía del Obispo, 38: Cayo Verde, 39: Pasa del Obispo, 40: Canalizo La Mahoma, 41: Canal de Ranchería, 42: Empalizada de Barlovento, 43: Cayo del Cristo, 44: Cayo Mariposa, 45: Cayo Dromedarios, 46: Cayo Lanzanillo, 47: Cayos de Pajonal, 48: Cayo Fragoso, 49: Cayo Francés, 50: Canalizo Chorros, 51: Canalizo Baliza Vieja, 52: Canalizo Baliza Nueva, 53: Cayo Guillermo, 54: Cayo Media Luna, 55 Cayo Coco, 56: Cayo Romano, 57: Cayo Anclitas, 58: Cayo Bretón.

Fig. 2. Distribution and relative abundance of Ecteinascidia turbinata, (\% of roots with ascidians) in Cuba.

entre 25 y 315 en Inés de Soto y entre 2 y 250 en Cayo Uvas. Los menores valores de biomasa se encontraron en Punta del Este, donde variaron entre 3 y $189 \mathrm{~g} / \mathrm{m}$, mientras que en las estaciones cercanas a Bahía de Cádiz estuvieron entre 20 y 250 . El promedio de la biomasa húmeda fue de $73.63 \mathrm{~g} / \mathrm{m}$.

No se encontraron diferencias significativas en la densidad de colonias entre las tres zonas de la plataforma cubana estudiadas, sin embargo al comparar las biomasas las diferencias sí fueron significativas $(\mathrm{p}<0.05, \mathrm{~F}=26.9)$.

\section{DISCUSIÓN}

E. turbinata tiene la capacidad de fijarse y crecer sobre cualquier objeto permanentemente sumergido en el mar. La especie ha sido encontrada sobre numerosos sustratos (Berrill 1932, Van Name 1945, Pèrés 1954, Millar 1962, Ramos et al. 1993), tubos de poliquetos, bolsas de plástico, algas como Caulerpa prolifera, hojas secas de fanerógamas marinas, e incluso sobre fondos arenosos (Bingham y Young 1991b, Carballo et al. 1997). En el Caribe, 

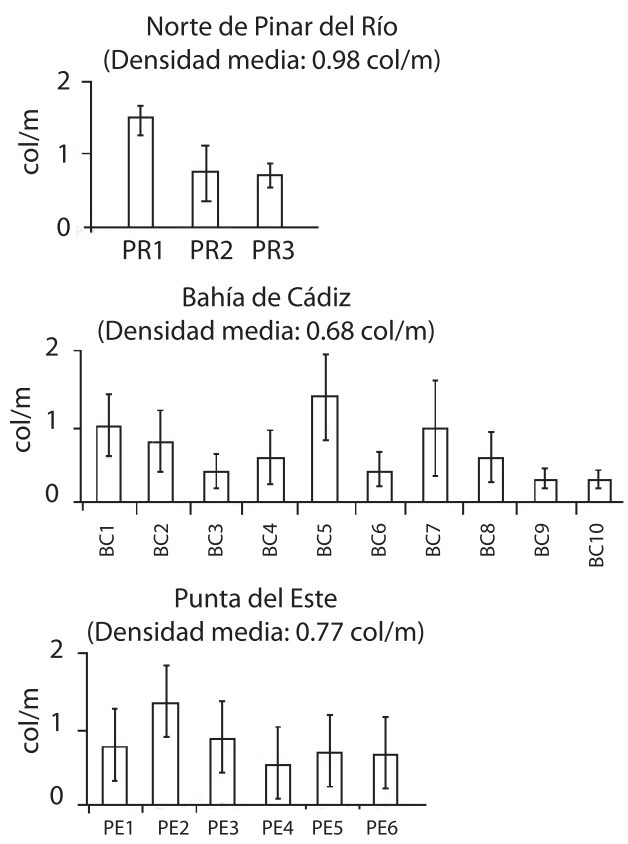

Fig. 3. Densidad de Ecteinascidia turbinata (número de colonias por metro de manglar recorrido) en tres localidades de las costas de Cuba: Punta del Este, Norte de Pinar del Río y Bahía de Cádiz (Valor promedio y desviación estándar). Fig. 3. Density of Ecteinascidia turbinata (number of colonies per meter of mangrove) in three locations of cuban coasts (A. Punta del Este, B. Norte de Pinar del Río y C. Bahía de Cádiz. Average and standard deviation). aunque también ocupa cualquier tipo de sustrato duro sumergido, su amplia distribución y elevada abundancia está estrechamente relacionada con la presencia de los manglares de cayo o sobrelavados, cuyo borde externo posea profundidades mayores de un metro. Este constituye el hábitat preferido de la especie pues el autorreclutamiento de sus poblaciones y la disponibilidad de nuevos sustratos con el crecimiento constante de las raíces ofrece el espacio suficiente para el crecimiento vegetativo de este organismo (Hernández-Zanuy et al. 2000). Por otra parte, esta situación sólo se encuentra en aquellas zonas del Atlántico, donde las amplitudes promedios de las mareas sean menores de un metro ya que de lo contrario la superficie de la raíz quedaría expuesta al aire provocando la muerte de E. turbinata y otros miembros de la epibiota asociada. Sólo se encuentran mareas de este tipo en las costas del Caribe (Spalding et al. 1997) y en el Mar Mediterráneo.

Los resultados de abundancia obtenidos en este estudio son similares a los encontrados por Carballo (2000a) en algunas localidades del SE de México. Las densidades medias fueron cercanas a una colonia por metro de manglar recorrido en ambas localidades, y las biomasas promedios en México (Yucatán) y Cuba fueron 115 y $73 \mathrm{~g} / \mathrm{m}$, respectivamente. En el

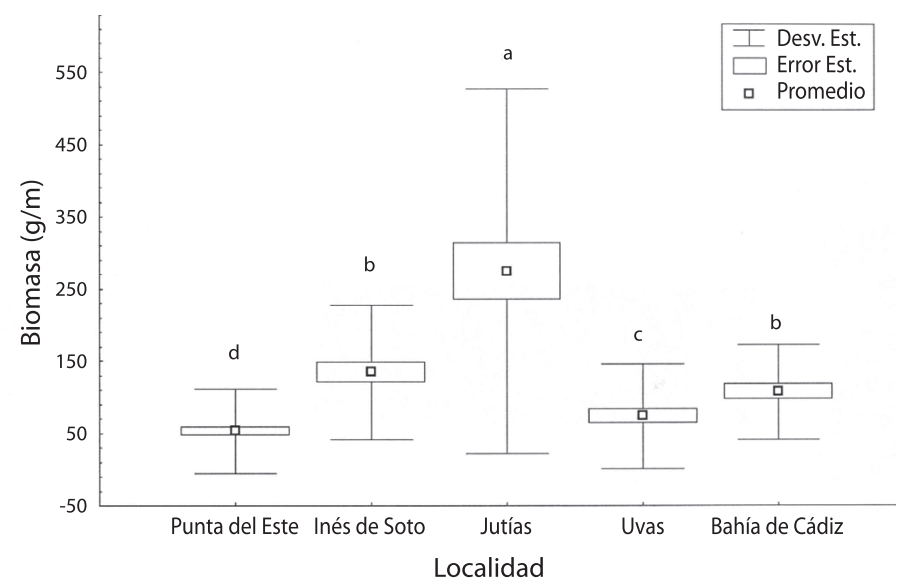

Fig. 4. Biomasa de Ecteinascidia turbinata (gramos por metro de manglar recorrido) en cinco localidades de las costas de Cuba: Punta del Este, Cayo Inés de Soto, Cayo Jutías, Cayo Uvas y Cayos Bahía de Cádiz (Valor promedio y desviación estándar; $\mathrm{F}=26.9, \mathrm{p}<0.05)$.

Fig. 4. Biomass of Ecteinascidia turbinata (gram per meter of mangrove) in five locations of Cuba: Punta del Este, Cayo Inés de Soto, Cayo Jutías, Cayo Uvas y Cayos Bahía de Cádiz (Average and standard deviation; F=26.9, p<0.05). 
caso de las poblaciones de E. turbinata en el Mediterráneo, donde el sustrato disponible se encuentra sobre el lecho marino, se encontraron biomasas entre 27 y $47 \mathrm{~g} / \mathrm{m}^{2}$ de fondo (Carballo et al. 1997). En el Mediterráneo existen poblaciones muy abundantes y efímeras que sólo se mantienen desde mayo hasta septiembre de cada año (Carballo et al. 1997, Carballo 2000b) en lagunas interiores de las costas europeas y africanas, como la Laguna de Formentera (Islas Baleares), Cádiz y Túnez, donde la especie ha sido capaz de dominar sobre todos los miembros de la comunidad (Ramos 1991). En el Caribe por el contrario, la presencia de la especie es permanente y su abundancia se mantiene todo el año, sólo con algunas fluctuaciones en dependencia de los períodos de reproducción y del ciclo de vida del animal. Se ha planteado que la temperatura óptima para la reproducción sexual de esta especie en el Caribe está entre 22 y $25{ }^{\circ} \mathrm{C}$ (Hernández-Zanuy et al. 2000) por lo que en el Mediterráneo la especie aprovecha al máximo los meses del año en los que la temperatura del agua se mantiene por encima de 20 ${ }^{\circ} \mathrm{C}$ para reproducirse y formar poblaciones muy numerosas con el fin de garantizar la supervivencia de los ejemplares del próximo año.

La elevada fragmentación de los cayos de mangle en numerosos canales, bordeados en ambas costas por Rhizophora mangle, supone una alta disponibilidad de sustrato para el asentamiento de E. turbinata. A pesar de no haber realizado prospecciones en algunas áreas del archipiélago cubano, tales como los cayos que se encuentran entre Cayo Buenavista y la Península de Guanahacabibes, es de suponer que la especie se encuentre también en dichas localidades por la similitud de condiciones hidrográficas en la zona. Sin embargo, en aquellas áreas de cayos cercanas a la desembocadura de ríos caudalosos o ciénagas, tales como la cuenca del Cauto, Río Zaza y la Ciénaga de Zapata, la especie está ausente. Es probable que los grandes volúmenes de agua dulce y sedimentos que en período de lluvia llegan al medio marino adyacente a estas zonas, provoquen cambios bruscos en la salinidad y sedimentación que impidan el desarrollo de E. turbinata. Por lo general en estas zonas el ostión de mangle Crassostrea rhizophorae ocupa el nicho ecológico sobre las raíces de mangle rojo sumergidas.

En Cuba, la gran extensión natural del ecosistema de manglar, segundo lugar de América Latina y primero de los países insulares (Lacerda 1993), y la ventaja de que el $57 \%$ de estos manglares son de tipo sobrelavado, donde el intercambio de agua con el océano mantiene más estables los parámetros ambientales más importantes para la especie, como la temperatura, la salinidad e inmersión permanente, hacen de esta región un área con potencial para la explotación racional de este recurso.

\section{AGRADECIMIENTOS}

El presente trabajo fue realizado gracias al financiamiento de la Agencia de Medio Ambiente del Ministerio de Ciencia y Tecnología de Cuba y la colaboración de la empresa española PharmaMar S.A.

\section{RESUMEN}

Ecteinascidia turbinata está ampliamente distribuida en las áreas de mangle rojo (Rhizophora mangle) de tipo sobrelavados donde ocupa la porción permanentemente sumergida. Ocasionalmente se encontró sobre el coral negro Antipathes caribeana a 22 y $38 \mathrm{~m}$ de profundidad. Sin embargo no fue frecuente en los manglares de borde ni de ribera. Se muestrearon 58 localidades estando presente la especie en un $75 \%$ de las mismas. Sus poblaciones son abundantes pues en el $57 \%$ de las zonas prospectadas más del $25 \%$ de las raíces del manglar poseen al menos una colonia. Las mayores densidades se encontraron en la costa noroeste de Cuba (Pinar del Río) con valores cercanos a una colonia por metro lineal de manglar, siendo Cayo Jutías la de mayor densidad $(1.46 \mathrm{col} / \mathrm{m})$. La mayor biomasa también se registró en la mencionada localidad con valores comprendidos entre 25 y $660 \mathrm{~g} / \mathrm{m}$ lineal de manglar. El promedio general de la biomasa en los manglares estudiados fue de $73.63 \mathrm{~g} / \mathrm{m}$ lineal de manglar.

Palabras clave: asidia, Ecteinascidia turbinata, distribución, abundancia, densidad, biomasa, manglar, Cuba. 


\section{REFERENCIAS}

Berrill, N.J. 1932. The mosaic development of the ascidian egg. Biol. Bull. 63:381-386.

Bingham, B.L. \& C.M. Young. 1991a. Influence of sponges on invertebrate recruitment: a field test of allelopathy. Mar. Biol. 109: 9-26.

Bingham, B.L. \& C.M. Young. 1991b. Larval behavior of the ascidian Ecteinascidia turbinata Herdman; an in situ experimental study of the effects of swimming on dispersal. J. Exp. Mar. Biol. Ecol. 145:189-204.

Bingham, B.L. \& C.M. Young. 1995. Stochastic events and dynamics of a mangrove root epifaunal community. PSZN I: Mar. Ecol. 16:145-163.

Carballo, J.L. 2000a. Distribución de Ecteinascidia turbinata (Ascidiacea: Perophoridae) en los manglares de la Península de Yucatán (México). Rev. Biol. Trop. 48:2-6.

Carballo, J.L. 2000b. Larval ecology of an ascidian tropical population in a mediterranean enclosed ecosystem. Mar. Ecol. Prog. Ser. 195:159-167.

Carballo, J.L., S. Naranjo, B. Kukurtzü \& A. HernándezZanuy. 1997. Estudio de la población de Ecteinascidia turbinata Herdman, 1880 (Ascidiacea:Perophoridae) en la isla de Formentera (Mar Mediterráneo. España): Distribución, densidad y crecimiento. Cien del Mar, UAS 15:7-15.

Carballo, J.L., A. Hernández-Zanuy, S. Naranjo, B. Kukurtzü \& A. García Cagide. 1999. Recovery of Ecteinascidia turbinata Herman, 1880 (Ascidiacea: Perophoridae) populations after different levels of harvesting on a sustainable basis. Bull. Mar. Sci. 65:755-760.

Carballo, J.L., S. Naranjo, B. Kukurtzü, F. de La Calle \& A. Hernández-Zanuy. 2000. Production of Ecteinascidia turbinata (Ascidiacea: Perophoridae) for obtaining anticancer compounds. J. World Aqua. Soc. 31:481-490.

García-Cagide A., A. Hernández-Zanuy \& A. Cárdenas. 2005. Fecundidad y primeras fases del desarrollo larval de la ascidia Ecteinascidia turbinata en Cuba. Bol. Invest. Mar. Cost. 34: 123-139.

Guan, Y., R. Sakai, K.L. Rinehart \& A.H. Wang. 1993. Molecular and crystal structures of ecteinascidins: potent antitumor compounds from the Caribbean tunicate Ecteinascidia turbinata. Biomol. Struct. Dyn. 10:793-818.
Hernández-Zanuy, A. 1990. Lista de ascidias de aguas cubanas. Poeyana 388:1-7.

Hernández-Zanuy A., A. García-Cagide, M. Esquivel \& A. Blanco. 2000. Reproducción y desarrollo de Ecteinascidia turbinata (Ascideacea: Perophoridae) en Cuba. Rev. Biol. Trop. 48:193-199.

Hernández-Zanuy A. 2003. Biología y ecología de Ecteinascidia turbinata Herdman, 1880 (Ascidiacea) en Cuba. Universidad de la Habana. La Habana, Cuba.

Kerr, R.G. \& N.F. Miranda. 1995. Biosynthetic studies of ecteinascidins in the marine tunicate Ecteinascidia turbinata. Nat. Prod. 58:1618-1621.

Lacerda, L. D. 1993. Mangroves of Latin America and the Caribbean: an overview. Proc. Workshop on Conservation and Sustainable Utilization of Mangroves Forests in Latin America and Africa Regions. ISME and ITTO. La Habana, Cuba.

Littler D. S. \& M. M. Littler. 1997. An illustrated marine flora of the Pelican Cays, Belize. Bull. Biol. Soc. of Washington. 9:1-149.

Lugo A. E. \& S.C. Snedaker. 1974. The ecology of mangroves. Ann. Rev. Ecol. Syst. 5: 39-64.

Millar, R. H. 1962. Further description of South African ascidians. Ann. S. Afr. Mus. 46: 1-221.

Millar, R. H. 1978. Ascidians of the Guyana. Shelf. Neth. J. Sea. Res., 12:99-106.

Monniot, F. 1972. Ascidies aplousobranches des Bermudes. Polyclinidae et Polycitoridae. Bull. Mus. Nat. Hist. 61: 949-962.

Monniot, C. \& F. Monniot. 1984. Ascidies littorales de Guadalupe. IX. Caracteristiques des populations, écologie, rapports avec la faune mondiale. Théthys 11 (3-4): 203-213.

Naranjo, S. A. 1995. Taxonomía, zoogeografía y ecología de las ascidias del Estrecho de Gibraltar. Implicaciones de su distribución bionómica en la caracterización ambiental de zonas costeras. Tesis Doctoral, Universidad de Sevilla. Sevilla, España.

Orihuela, B. \& H. Díaz. 1991. Mass mortality in a mangrove roots fouling community in a hypersaline tropical lagoon. Biotropica 23(4): 592-601.

Pain, S. 1996. Hostages of the deep. New Sci. 151:38-42.

Pèrés, J.M. 1954. Contribution à l'etude des ascidies de Tunisie. Bull. Stn. Océanogr. Slambo. 49: 3-21. 
Pèrés, J.M. 1956. Etudes sur l'Illot du Grant Congloué VII. Ascidies. Ann. Inst. Océanogr. París. 32: 231-232.

Ramos, A. 1991. Ascidias litorales del Mediterráneo Ibérico. Faunística, ecología y biogeografía. Tesis de Doctorado. Universidad de Alicante, España. $425 \mathrm{p}$.

Ramos, A., V. Buencuerpo, E. Vazquez \& F. Lafarque. 1993. Distribución bionómica de las ascidias litorales del Estrecho de Gibraltar. Publ. Espec. Inst. Esp. Oceanogr. 11:2-36.

Rinehart, K.H., T.G. Holt, N.L. Fregeau, J.G. Stroh, P.A. Keiper, F. Sun, L.H. Li \& D.G. Martín. 1990. Ecteinascidins 729, 743, 745, 579A, 759B and 770. Potent antitumor agents from the Caribbean tunicate Ecteinascidia turbinata. Jour. Org. Chem. 55: 452-455.

Saito, N., K. Tashiro, Y. Maru, K.Yamaguchi \& A. Kubo. 1997. Synthetic approaches toward ecteinascidins .1. Preparation of an (E)-2-arylidene-3-benzyl-1,5- imino-3-benzazocin-4-one having a protected phenol in the E-ring. Jour. Chem. Soc. Perkin Tr. 1:53-69.

Sakai, R., K.L. Rinehart, Y. Guan \& A.H. Wang. 1992. Additional antitumor ecteinascidins from a Caribbean tunicate: crystal structures and activities in vivo. Proc. Nat. Acad. Sci. USA 89:11456-11460.

Sakai, R., K.L. Rinehart, Y. Guan \& A.H. Wang. 1996. Ecteinascidins: putative biosynthetic precursors and absolute stereochimistry. Jour. Amer. Chemm. Soc. 118:9017-9023.

Spalding, M. D., Blasco, F. \& Field, C. D1997. World Mangrove Atlas. The International Society for Mangrove Ecosystems, Okinawa, Japan. 178 p.

Van Name, W.G. 1921. Ascidians of the West Indian region and southern United States. Bull. Amer. Mus. Nat. Hist. Vol XLIV:283-494.

Van Name, W. G. 1945. The North and South American Ascidians. Bull. Am. Mus. Nat. Hist. 84:1-476. 